OPEN ACCESS

Edited by: Shengxiang Ren, Tongji University, China

Reviewed by:

Xuefei Li,

Shanghai Pulmonary Hospital, China Anastasios Dimou, Mayo Clinic, United States

*Correspondence:

Xiao-Juan Chen

366535255@qq.com

${ }^{\dagger}$ These authors have contributed equally to this work and share first authorship

Specialty section: This article was submitted to

Cancer Immunity and Immunotherapy, a section of the journal Frontiers in Immunology

Received: 06 February 2021 Accepted: 29 March 2021 Published: 19 April 2021

Citation:

Chen X-J, Ren A-Q, Zheng L and Zheng E-D (2021)

Predictive Value of KDM5C Alterations for Immune Checkpoint Inhibitors Treatment Outcomes in Patients With Cancer.

Front. Immunol. 12:664847. doi: 10.3389/fimmu.2021.664847

\section{Predictive Value of KDM5C Alterations for Immune Checkpoint Inhibitors Treatment Outcomes in Patients With Cancer}

\author{
Xiao-Juan Chen ${ }^{1,2 * t}$, Ai-Qun Ren ${ }^{2 \dagger}$, Liang Zheng ${ }^{2 \dagger}$ and En-Dian Zheng ${ }^{2 \dagger}$ \\ ${ }^{1}$ Department of Clinical Medicine, Graduate School, Zhejiang Chinese Medical University, Hangzhou, China, ${ }^{2}$ Department of \\ Gastroenterology, Wenzhou People's Hospital, Wenzhou Third Clinical Institute Affiliated to Wenzhou Medical University, \\ Wenzhou, China
}

Lysine (K)-specific demethylase 5C (KDM5C) plays a significant role in the tumor cell proliferation, invasion, drug resistance and the regulation of tumor-related gene expression. Here, we aimed to investigate its predictive value in patients with cancers received immune checkpoint inhibitors (ICls). We explored the predictive value of KDM5C alterations and the association between $K D M 5 C$ alteration and immune landscape by using published cohort with clinical outcome and sequenced data from online database. The frequency of KDM5C alterations was $2.1 \%$ across 48045 tumor samples with different cancers from 185 studies. KDM5C alterations were correlated with markedly inferior overall survival (OS, 53 vs. 102 months, $P<0.0001$ ) than those without. However, in $\mathrm{ICl}$-treated group, patients with $K D M 5 \mathrm{C}$ alterations had a substantially prolonged OS than the wild-type group (not reached vs. 18 months, $P=0.0041$ ). The predictive value of KDM5C alterations for $\mathrm{ICl}$ treatment outcome was not observed in patients with microsatellite-stable tumors $(P=0.2875)$. Intriguingly, patients with non-small-cell lung cancer and $K D M 5 C$ alterations receiving $\mathrm{ICl}$ had the better progression-free survival than wild type group (13.2 vs. 3.2 months, $P=0.0762$ ). Mechanistically, KDM5C altered tumors had dramatically higher TMB level and was associated with significantly higher level of CD8+ $\mathrm{T}$ cell infiltration and $\mathrm{T}$ effector signature. In conclusion, KDM5C alterations was correlated with enhanced tumor immunogenicity and inflamed anti-tumor immunity, thus resulting in better treatment outcome in cancer patients receiving ICls.

Keywords: immune checkpoint inhibitors, biomarker, KDM5C, outcome, prognosis

\section{INTRODUCTION}

Immune checkpoint inhibitors (ICIs) targeting cytotoxic T lymphocyte antigen-4 (CTLA-4), or programmed cell death protein 1 (PD-1) and its ligand (PD-L1) interaction have shifted the treatment paradigms and significantly improve the overall survival (OS) in diverse cancers (1-4). Nevertheless, ICIs could only benefit a minority $(20 \%)$ of unselected population (5). Herein, there is an urgent need to develop novel predictive biomarkers for the majority of patients, who could not benefit from ICIs treatment. The mutational landscape of tumor cells is a direct reflection of tumor 
immunogenicity and could dictate the extent and phenotype of immune infiltrates (6-8). Understanding the relationship between tumor genomic alterations and response to ICIs could lay a foundation for the development of novel predictive biomarkers and therapeutic strategies to improve the clinical benefit (8).

Lysine $(\mathrm{K})$-specific demethylase $5 \mathrm{C}(K D M 5 C)$ is a histone demethylase that specifically removes methyl residues from tri-, di-, and monomethylated lysine 4 on histone H3 lysine 4 (H3K4), thus resulting in suppressing gene transcription by reducing $\mathrm{H} 3 \mathrm{~K} 4$ trimethylation levels (9-11). Previous studies reported that genetic alterations of $K D M 5 C$ were common in various types of cancers including breast, colon, ovarian, prostate cancer and so on. It plays a significant role in the tumorigenesis, cancer cell proliferation, invasion, drug resistance and the regulation of tumor-related gene expression (12-14). Moreover, a recent elegant study analyzed the multi-omics data of 823 advanced renal cell carcinoma and found that somatic mutations in KDM5C correlate with high angiogenesis and AMPK/fatty acid oxidation gene expression, which was enriched in ICIs beneficial group. These findings revealed the contribution of $K D M 5 C$ to antitumor immune response. Therefore, it is valuable to explore the predictive value of $\mathrm{KDM} 5 \mathrm{C}$ alterations for ICIs treatment outcome in multiple cancers.

Here, we performed this pan-cancer analysis to investigate KDM5C alterations frequency and their predictive significance for ICIs treatment outcomes across cancer types. We also evaluated the relationship between $K D M 5 C$ alteration and immune infiltrates and signatures by using online database to unravel the potential mechanism.

\section{MATERIALS AND METHODS}

\section{Data Collection and Pan-Cancer Analysis}

We downloaded the sequenced data and collected clinical information from several online database as shown in Figure 1. For determination of the frequency of KDM5C alterations among different types of solid tumors, the genomic alterations and clinical characteristics were identified from the cBioPortal online database (https://www.cbioportal.org) $(15,16)$. KDM5C alterations were recorded as all kinds of nonsynonymous mutations including mutations, missense, frame-shift, splice site, nonstop, nonsense, and translation start site changes. Non-redundant publications were identified. If two or more studies reported the same cohort, only the study with the largest sample size and latest information was included. To avoid the selection bias and limitation of small sample size, we excluded the records of cancer type with patients less than 100 . Analysis of TMB normalization, clinical cohort and treatment outcomes were summarized in Supplemental Material.

\section{TUMOR IMMUNOGENICITY AND IMMUNE LANDSCAPE ANALYSIS}

To delineate the immune microenvironment features of tumors with $K D M 5 C$ alterations, we calculated and compared immune infiltrates, immune signatures and immune-related gene expression between $\mathrm{KDM} 5 \mathrm{C}$ altered and wild type group by using RNA-seq data from TCGA. The correlation between

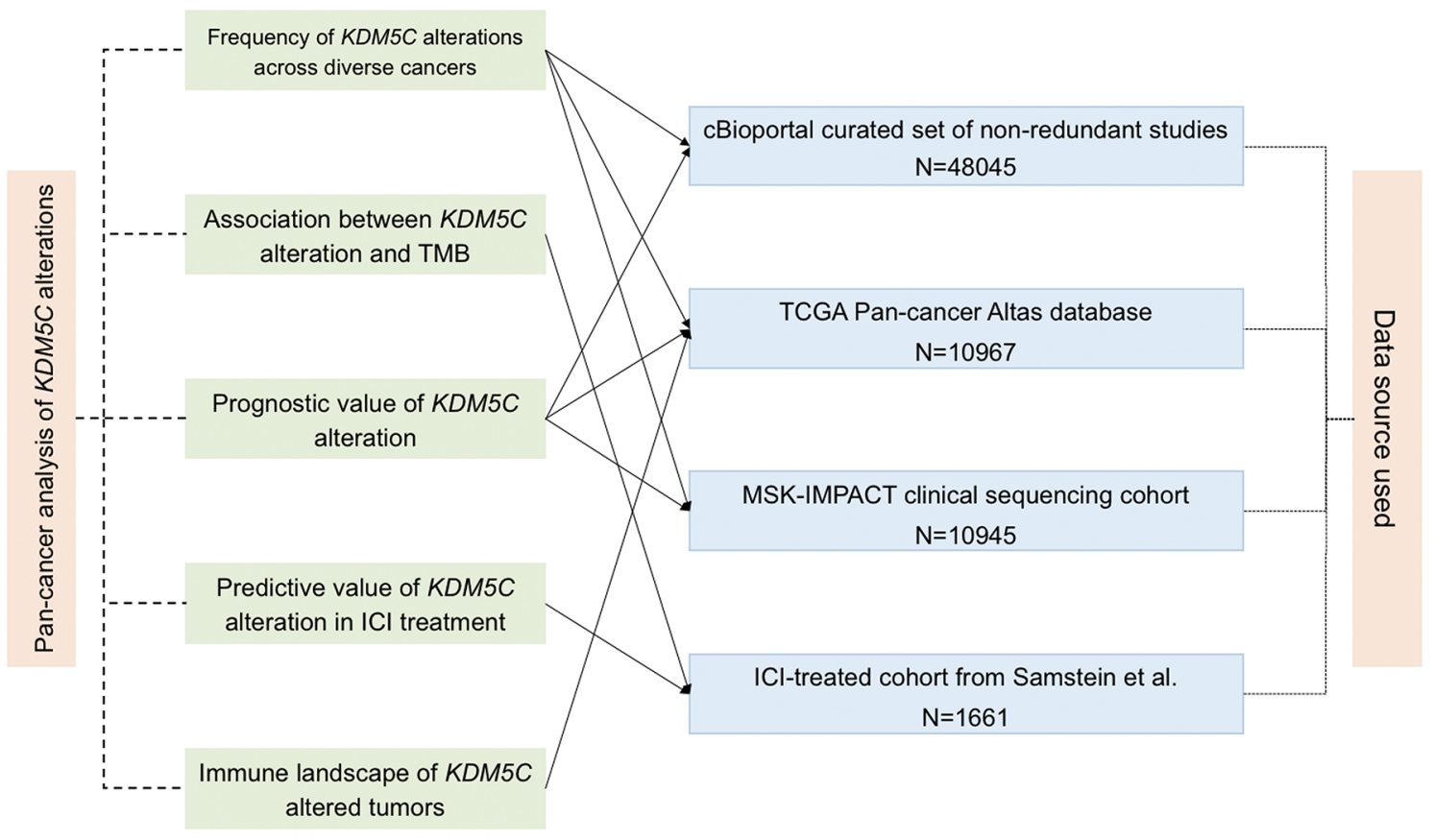

FIGURE 1 | Flowchart of the sequenced data and clinical cohort. The connected solid line between analysis aim (middle left) and data source (middle right) means the used cohort by this analysis. 
KDM5C expression and immune checkpoints expression in different cancers was evaluated by using online database, named Tumor Immune Estimation Resource (TIMER). The statistical methods were listed in this website (https://cistrome. shinyapps.io/timer/) and their previous publications $(17,18)$. The abundance of tumor infiltrating leukocytes, including $\mathrm{CD} 8^{+}$ $\mathrm{T}$ cells, $\mathrm{CD} 4^{+} \mathrm{T}$ cells, regulatory $\mathrm{T}$ cells (Tregs), dendritic cells, B cells, macrophage, myeloid-derived suppressor cells (MDSC), NK cells, mast cell, neutrophils, endothelial cells and cancerassociated fibroblasts (CAFs), was estimated by using different bioinformatic algorithm and compared between $K D M 5 C$ altered and wild type group.

\section{Statistical Analysis}

The association between KDM5C status and clinical features were evaluated by using fisher's exact test. $\chi 2$ test were performed to test whether the sampling distribution was equal for two groups. The continuous variables were analyzed by ANOVA and Tukey's multiple comparison tests. The differences of TMB, tumor-infiltrating immune cells, immune signatures, or immune-related gene expressions between KDM5C altered and wild type tumors were tested by using Mann-Whitney U test. We conducted two-tailed MannWhitney $\mathrm{U}$ tests for comparison of the nonparametric data set. Survival outcomes were measured with OS, or progression-free survival (PFS) according to the accessibility for each cohort. Kaplan-Meier curves with two-sided log-rank tests and Cox proportional hazards model with calculated hazard ratios (HRs) and 95\% confidence intervals (CIs) were adjusted for available confounding factors to determine the different clinical outcomes between KDM5C altered and wild-type groups. Twosided $P<0.05$ was considered significant. All statistical analyses were performed using the SPSS statistical software, version 20.0 (SPSS Inc., Chicago, IL, USA).

\section{RESULTS}

\section{Overview of Pan-Cancer Analysis}

We identified a cohort of 45614 cancer patients with 48045 sequenced tumor samples. This cohort was consisted of 271 cancer studies and 47 cancer types. The prevalence of KDM5C alterations was $2.1 \%$, with patients with esophagogastric cancer having the highest levels of KDM5C alterations (11.5\%, 118/ 1023). We then investigated the prevalence and spectrum of KDM5C alterations in two representative cohorts (TCGA cohort, $\mathrm{N}=10967$; MSK-IMPACT cohort, $\mathrm{N}=10945$ ). In TCGA cohort, endometrial carcinoma had the highest levels of KDM5C alterations (9.6\%, 56/586; Figure 2A). In MSK-IMPACT cohort, renal cell carcinoma had the highest levels of $K D M 5 C$ alterations $(9.4 \%, 34 / 361$; Figure 2B). Most detected KDM5C alterations were copy number alterations (either amplifications or deep deletion) in TCGA cohort (Figure 2A), while most were KDM5C somatic mutations in MSK-IMPACT cohort (Figure 2B).

\section{Association Between KDM5C Alterations and Clinical Outcomes}

Next, we evaluated the association between KDM5C alterations and clinical outcomes. We firstly found that patients with KDM5C alterations showed a significantly shorter OS (53 vs. 102 months; $\mathrm{HR}=1.31$, 95\% CI 1.17-1.58, $P<0.0001$; Figure 3A) than those without in 45614 cancer patients by merging 271 non-redundant studies from the cBioPortal online database. Subgroup analyses showed that KDM5C alterations were correlated with numerically shorter OS in TCGA (68 vs. 80 months; $P=0.4336$; Figure 3B) and MSK-IMPACT cohort (23 vs. 26 months, $P=0.5220$; Figure $3 C$ ).

In the ICI treatment cohort (19), we firstly identified 1661 patients with different cancers receiving ICI therapy and 73 of
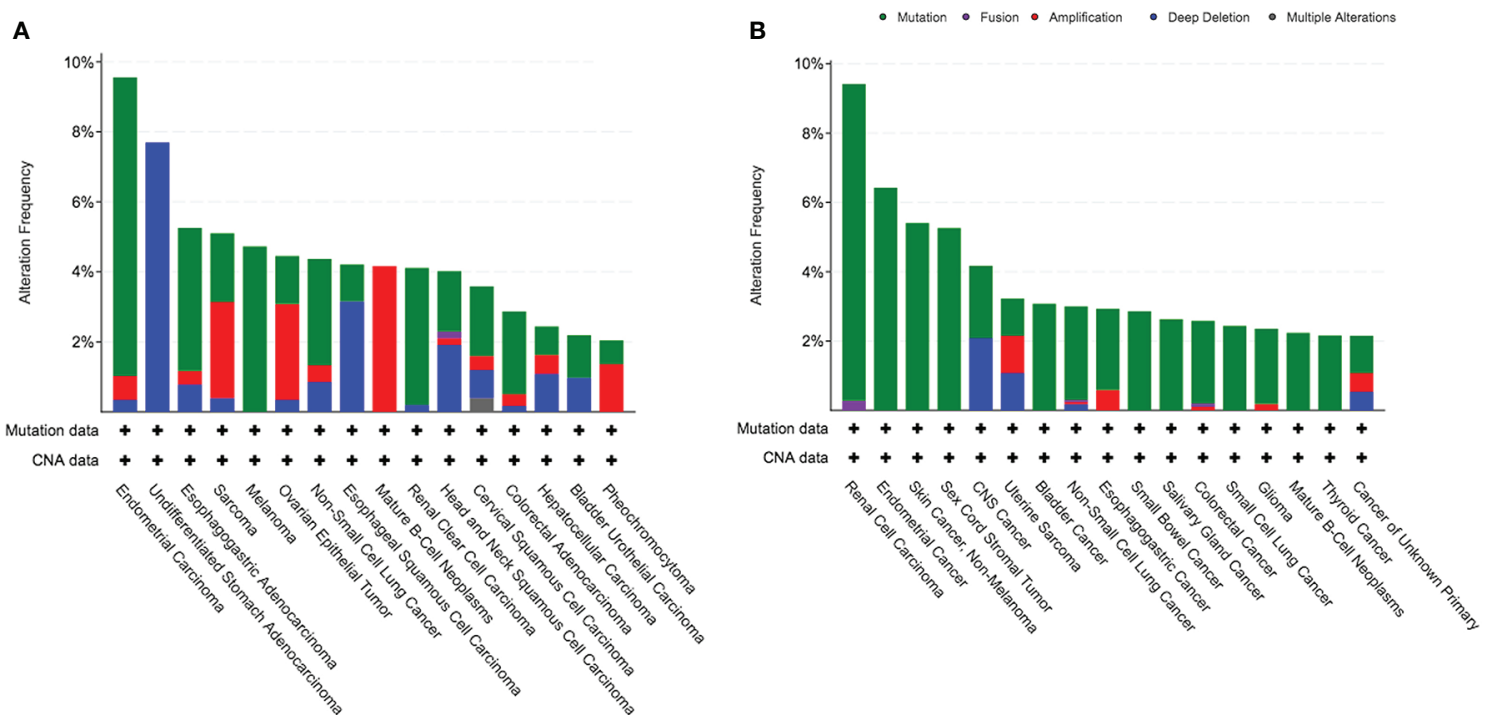

FIGURE 2 | Prevalence of KDM5C alterations in different cancers. (A) TCGA cohort; (B) MSK-IMPACT cohort. 
them with $K D M 5 C$ alterations. Clinicopathological features, including age, sex, sample type, drug type and tumor purity, were well balanced between altered and wild type group (Supplemental Table S1). Patients with KDM5C alterations had a significantly prolonged OS than those in wild-type group (not reached vs. 18 months; $\mathrm{HR}=0.56,95 \% \mathrm{CI} 0.46-0.86, P=$ 0.0041; Figure 3D). Importantly, we compared the overall survival of patients who received ICI with those who did not in $\mathrm{KDM}$ 5C mutant group. As shown in the following figure $\mathrm{A}$, we found that patients received ICI treatment had markedly longer overall survival than those received chemotherapy in KDM5C mutant group $(\mathrm{HR}=0.584, \mathrm{P}=0.0168$; Supplemental Figure S2A). However, in KDM5C wild type group, patients received ICI treatment had analogous overall survival with those received chemotherapy $(\mathrm{HR}=0.949, \mathrm{P}=0.1067$; Supplemental Figure S2B). Although $K D M 5 C$ alterations were associated with higher level of TMB and mutation count, multivariate analysis revealed that $K D M 5 C$ alterations was associated with substantially longer OS than wild type independent of TMB (HR $=0.60,95 \%$ CI 0.40 0.91, $P=0.015$; Supplemental Table S2). Notably, we did not observe the association between KDM5C alterations and better OS in patients with microsatellite-stable (MSS) solid tumors (12 vs. 21 months; $\mathrm{HR}=1.62,95 \% \mathrm{CI} 0.50-5.63, P=0.2875$; Figure 3E). Interestingly, in non-small-cell lung cancer (NSCLC) treated with ICI, patients with KDM5C alterations had markedly longer progression-free survival (PFS) than other alterations and wild type groups (13.2 vs. 3.2 months; $\mathrm{HR}=$ $0.52,95 \%$ CI $0.34-1.05, P=0.2875 ; P=0.0762$; Figure 3F).

\section{Association Between KDM5C Alteration and TMB Level}

Previous publications revealed the close relationship between ICIs treatment outcomes and $\mathrm{TMB} /$ mutation counts. Thus, it is valuable to evaluate the relationship between $K D M 5 C$ alterations and TMB level/mutation counts. In MSK-IMPACT cohort (20), we found that mutation count of patients with KDM5C alterations was significantly higher than those without these alterations (10 vs. $4, P<0.0001$; Supplemental Figure S1). This was validated in the ICI-treated cohort that included 1661 patients (mutation count of $K D M 5 C$ alterations vs. wild type: 15 vs. $6, P<0.0001$; Figure $4 \mathrm{~A}$ ). Notably, cancers with $K D M 5 C$ alterations also had the higher TMB level than those without these alterations ( 12 vs. 6 mut/Mb, $P<0.0001$; Figure 4B). Cooccurring of genetic mutations in cancers with KDM5C alterations were not uncommon in both early-stage and advanced stage cohort (Figures $4 \mathrm{C}, \mathrm{D}$ ) and some of them are prevalent driver genes (e.g., LRP2, KMT2C, PBRM1, NOTCH1, FAT1, SETD2, NSD1, etc.), while their clinical significance remained undetermined.

\section{Immune Feature Analysis of KDM5C Altered Tumors}

To depict the tumor immune microenvironment of KDM5C altered tumors, we compared the immune infiltrates and antitumor immunity between $K D M 5 C$ altered and wild type tumors. As we previously mentioned, KDM5C altered tumors had significantly higher TMB level than those with wild type,
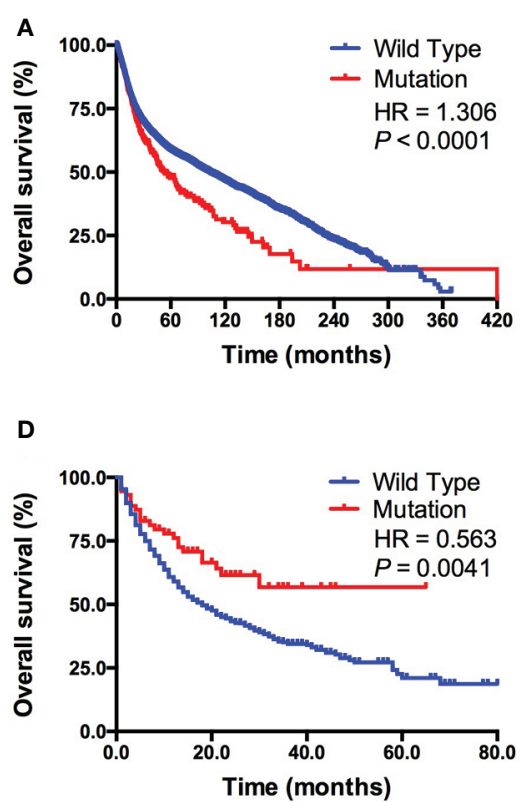
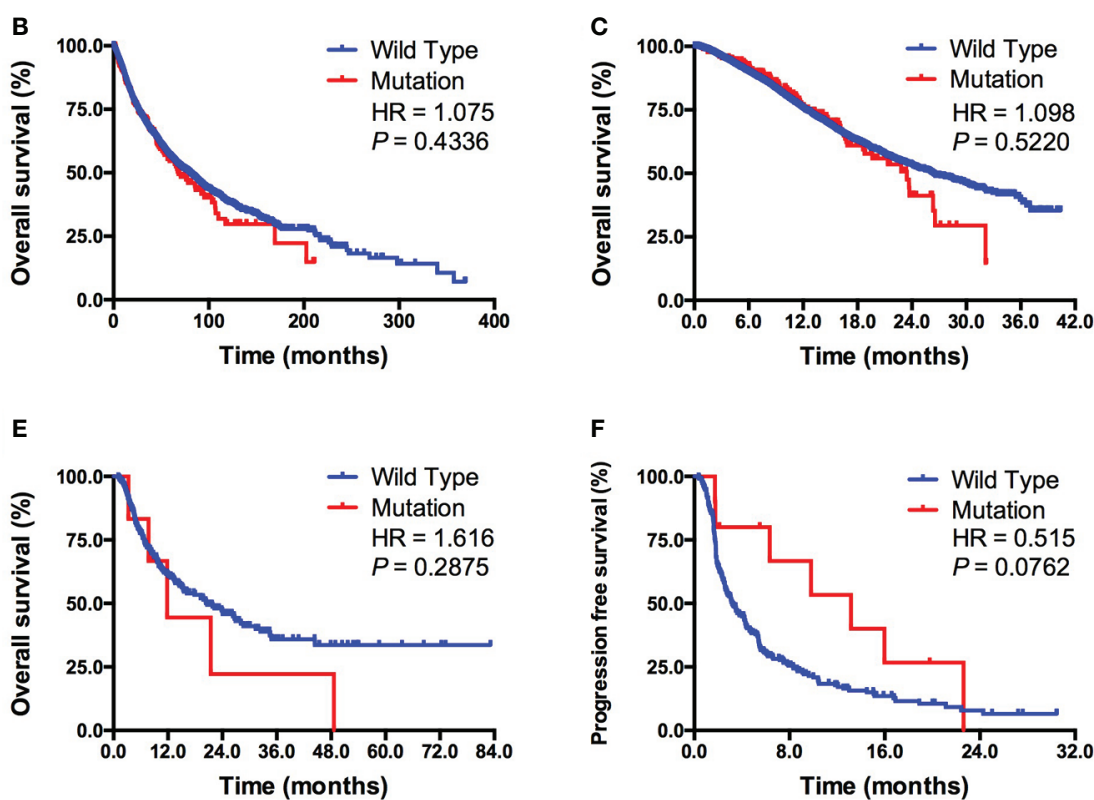

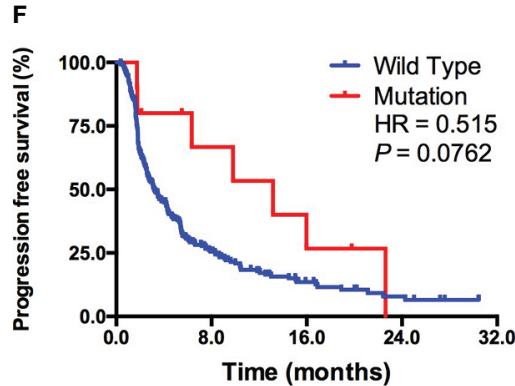

FIGURE 3 | Association between KDM5C alterations and clinical outcome. (A) Prognostic value of $K D M 5 C$ alterations in all cancers; (B) Prognostic value of $K D M 5 C$ alterations in TCGA cohort; (C) Prognostic value of KDM5C alterations in MSK-IMPACT cohort; (D) Predictive value of KDM5C alterations in ICI treated cohort; (E) Predictive value of KDM5C alterations in patients with microsatellite-stable solid tumors; (F) Predictive value of $K D M 5 C$ alterations in patients with non-small-cell lung cancer patients received $\mathrm{ICI}$ treatment. 


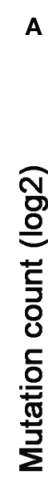

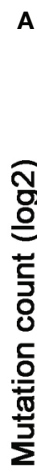

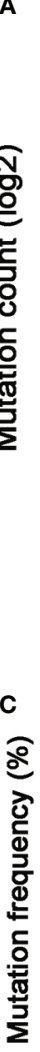

B
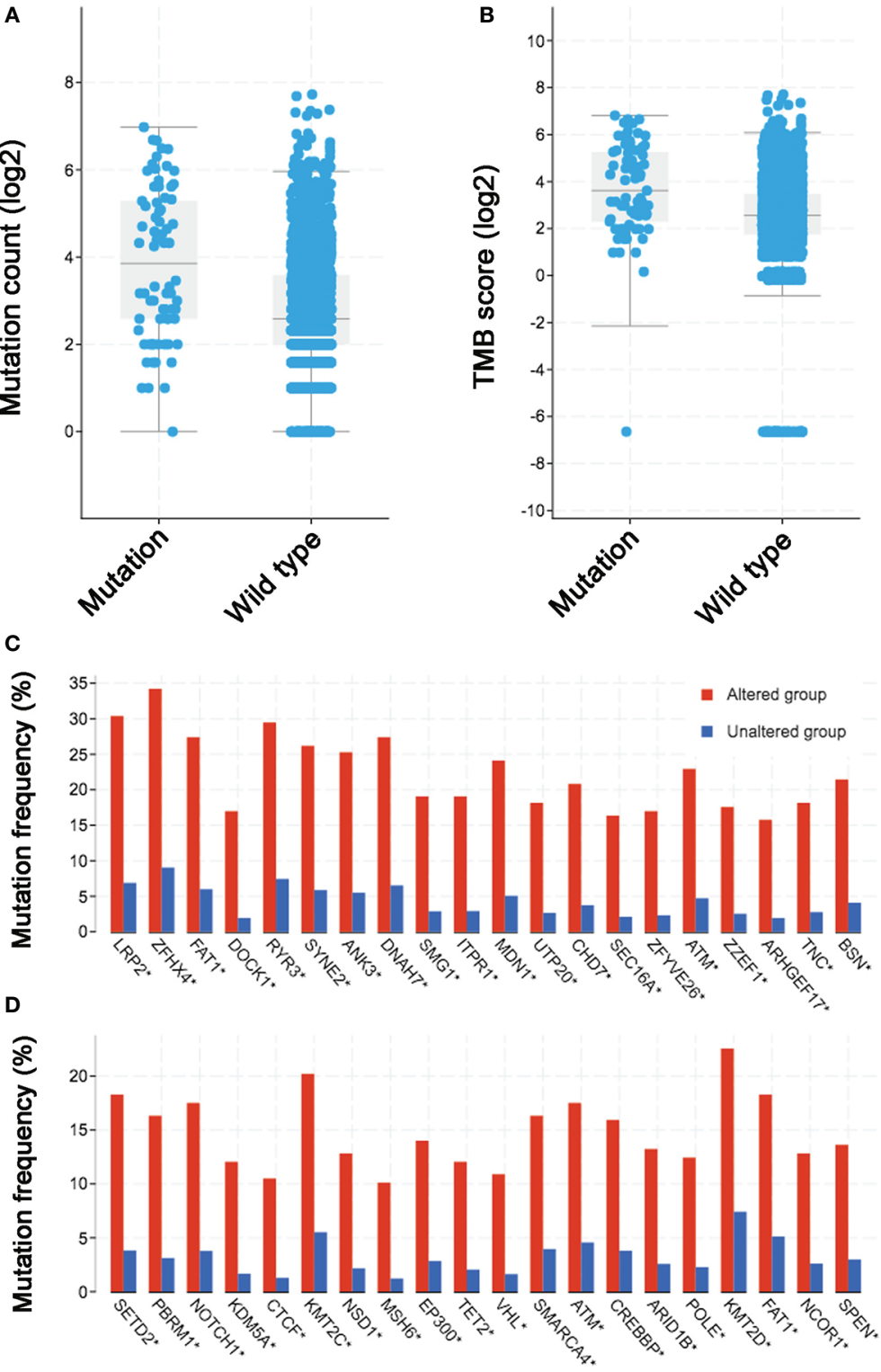

FIGURE 4 | Association between KDM5C alterations and mutation count/tumor mutation burden (TMB) across diverse types of cancer. (A) The association between mutation count and KDM5C alterations in immune checkpoint inhibitor (ICI) treated cohort; (B) The association between TMB and KDM5C alterations in ICI treated cohort; (C) Co-occurring of genetic mutations in cancers with KDM5C alterations versus wild type in TCGA cohort; (D) Co-occurring of genetic mutations in cancers with KDM5C alterations versus wild type in MSK-IMPACT cohort.

suggesting the potential enhanced tumor immunogenicity of KDM5C altered tumors. We then surveyed the relationship between $K D M 5 C$ alterations and common immune infiltrates including $\mathrm{CD}^{+} \mathrm{T}$ cells, $\mathrm{CD} 4^{+} \mathrm{T}$ cells, Tregs, dendritic cells, B cells, macrophage, MDSC, NK cells, mast cell, neutrophils, endothelial cells and CAFs across different cancer types (Figure 5 and Supplemental Figures S3-S8). The results showed that tumor-infiltrating $\mathrm{CD}^{+} \mathrm{T}$ cells, were generally more abundant in the KDM5C altered colon adenocarcinoma and uterine corpus endometrial carcinoma when compared with those in the wild type tumors (Figure 5A). Whereas other immune infiltrates had similar abundance in KDM5C altered and wild type group (Supplemental Figures S3-S8). Moreover, KDM5C altered colon adenocarcinoma and uterine corpus endometrial carcinoma had dramatically higher level of antitumor $\mathrm{T}$ effector signature (Figure 5B). We also evaluated the association between KDM5C expression and several inhibitory (e.g., CD160, CD96, CSF1R, CTLA-4, TIM-3, IDO1, IL10, LAG3, PD-1, PD-L1, PD-L2, TFGB1, TGFBR1, TIGIT, VEGFA) and stimulatory (e.g., CD27, CD28, CD40, CD40LG, CD70, CD80, CD86, CXCL12, CXCR4, ICOS, ICOSLG, MICA, MICB, TNFRSF14, TNFRSF17, TNFRSF18, TNFRSF4, 


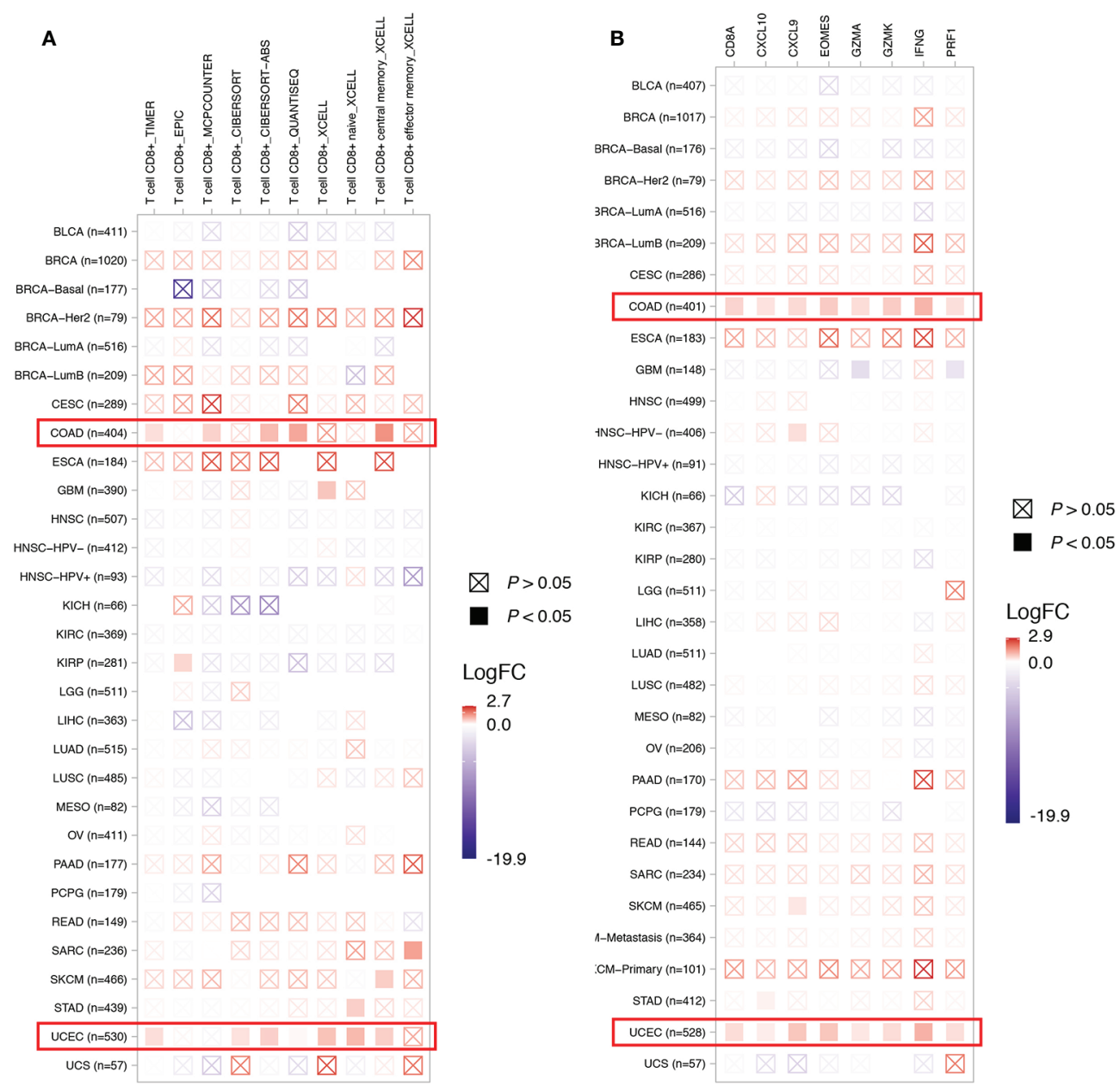

FIGURE 5 | Association between KDM5C alterations and CD8+ T cell abundance (A) and T effector signature (B). BLCA, Bladder Urothelial Carcinoma; BRCA, Breast invasive carcinoma; CESC, Cervical squamous-cell carcinoma and endocervical adenocarcinoma; COAD, Colon adenocarcinoma; ESCA, esophageal carcinoma; GBM, Glioblastoma multiforme; HNSC, head and neck squamous cell carcinoma; KICH, kidney chromophobe; KIRC, kidney renal clear cell carcinoma; KIRP, kidney renal papillary cell carcinoma; LIHC, Liver hepatocellular carcinoma; LUAD, lung adenocarcinoma; LUSC, lung squamouscell carcinoma; MESO, mesothelioma; OV, ovarian serous cystadenocarcinoma; PAAD, pancreatic adenocarcinoma; PCPG, pheochromocytoma and paraganglioma; READ, rectum adenocarcinoma; SKCM, Skin cutaneous melanoma; STAD, Stomach adenocarcinoma; UCEC, Uterine corpus endometrial carcinoma; UCS, uterine carcinosarcoma.

TNFRSF9, TNFSF9, TNFSF13) immune checkpoints expression in various cancers. Intriguingly, we also found the significantly higher expression level of these immune checkpoints in KDM5C altered colon adenocarcinoma and uterine corpus endometrial carcinoma (Figures 6A, B).

\section{DISCUSSION}

To our knowledge, this study firstly reported the frequency of $K D M 5 C$ alterations and its pan-cancer predictive value to ICI treatment in various cancers. KDM5C alterations were a negative prognostic marker in whole group but it might be utilized to predict survival benefit from ICI treatment across diverse cancers. Although $K D M 5 C$ altered tumors had significantly higher TMB level, multivariate analysis showed that $K D M 5 C$ alterations was associated with significantly longer OS independent of TMB. Moreover, we did not observe the association between KDM5C alterations and prolonged OS in patients with MSS solid tumors, suggesting that it may not be suitable for predicting ICI treatment outcome in MSS solid tumors. Mechanistically, KDM5C altered tumors was found to be markedly correlated with enhanced tumor immunogenicity and immunosupportive features of anti-tumor microenvironment.

In this pan-cancer analysis, the frequency of $K D M 5 C$ alterations was $2.1 \%$ in a cohort of 45614 cancer patients, with esophagogastric cancer, endometrial carcinoma and renal cell carcinoma having the highest levels of $K D M 5 C$ alterations, which was similar to previous publications $(21,22)$. Interestingly, we found a positive association between co-occurrence of $\mathrm{KDM} 5 \mathrm{C}$ alterations and some common 

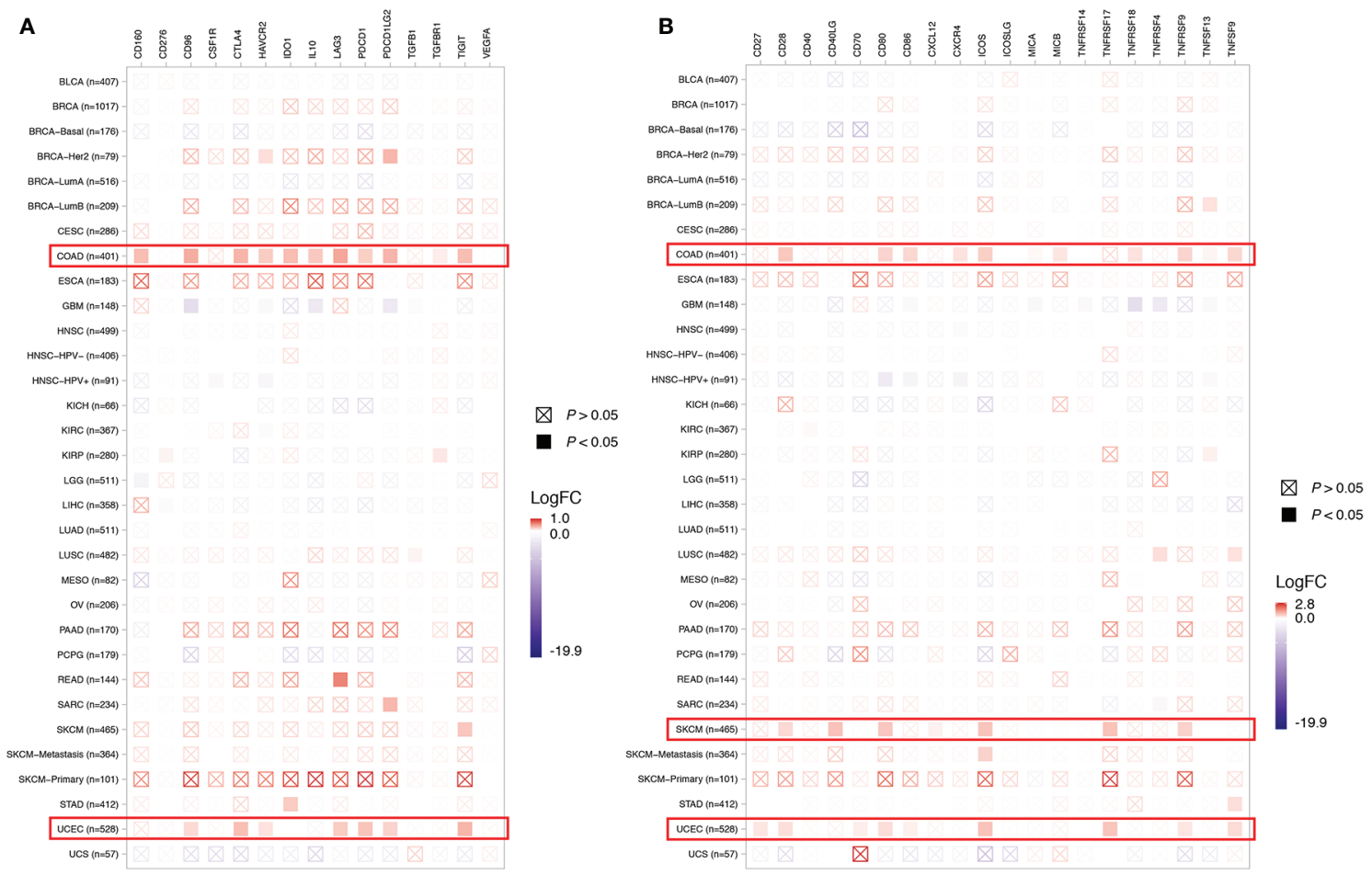

FIGURE 6 Association between KDM5C alterations and several inhibitory (A) and stimulatory (B) immune checkpoints expression across different cancer types. BLCA, Bladder Urothelial Carcinoma; BRCA, Breast invasive carcinoma; CESC, Cervical squamous-cell carcinoma and endocervical adenocarcinoma; COAD, Colon adenocarcinoma; ESCA, esophageal carcinoma; GBM, Glioblastoma multiforme; HNSC, head and neck squamous cell carcinoma; KICH, kidney chromophobe; KIRC, kidney renal clear cell carcinoma; KIRP, kidney renal papillary cell carcinoma; LIHC, Liver hepatocellular carcinoma; LUAD, lung adenocarcinoma; LUSC, lung squamous-cell carcinoma; MESO, mesothelioma; OV, ovarian serous cystadenocarcinoma; PAAD, pancreatic adenocarcinoma; PCPG, pheochromocytoma and paraganglioma; READ, rectum adenocarcinoma; SKCM, Skin cutaneous melanoma; STAD, Stomach adenocarcinoma; UCEC, Uterine corpus endometrial carcinoma; UCS, uterine carcinosarcoma.

epigenetic regulatory genes including PBRM1, KMT2C, SETD2, $N S D 1$, etc. In spite of the unclear biological function of these comutations, several previous studies have shown that these diver genes are very important tumor suppressor genes in renal cell cancers and could contribute to the aggressive phenotype, therapeutic efficacy and/or prognostic value (23-29). Therefore, it would be valuable to unravel the biological and molecular mechanisms, and impact on clinical outcome of this cooccurrence for specific cancer types in future studies.

Previous studies reported $K D M 5 C$ is required for proper DNA replication at early origins and its alterations could lead to genomic instability in sporadic renal cancer $(30,31)$. We thus evaluated the association between KDM5C alteration and TMB level. As expected, our results showed that KDM5C altered tumors had significantly higher TMB level than wild type ones in two independent cohorts, indicating that KDM5C alterations could be considered as predictive biomarkers for ICI treatment. Having noticed this relationship, we then investigated both predictive and prognostic significance of KDM5C alterations. In whole group, patients with $K D M 5 C$ alterations had a significantly shorter OS than those with wild type, suggesting that KDM5C alterations could not confer an intrinsic survival benefit to treatment-naïve patients receiving ICI treatment. In
ICI-treated cohort, patients with $K D M 5 C$ alterations had a substantially prolonged OS. Moreover, subgroup analyses showed the association between KDM5C alterations and OS was independent of TMB in patients receiving ICI. More interestingly, in NSCLC treated with ICI, we found patients with $K D M 5 C$ alterations had the significantly longer PFS than wild type groups. Collectively, KDM5C alterations could be considered as a potential pan-cancer predictive biomarker for ICI treatment, especially for NSCLC.

As a histone demethylase, KDM5C could suppress gene transcription by reducing $\mathrm{H} 3 \mathrm{~K} 4$ trimethylation levels (9-11). KDM5C plays a significant role in the tumorigenesis, cancer cell proliferation, invasion, metastasis and drug resistance (12-14). Recently, an elegant study analyzed the multi-omics data of 823 advanced renal cell carcinoma and found that somatic mutations in $K D M 5 C$ correlate with high angiogenesis and AMPK/fatty acid oxidation gene expression, which was enriched in ICIs beneficial group (32). These findings suggested that KDM5C altered tumor would have specific immune microenvironment features. In this study, we observed that $K D M 5 C$ altered tumors had markedly higher TMB and were associated with antiantitumor immune signatures, indicating that $K D M 5 C$ altered tumors would possess the enhanced tumor immunogenicity and 
relatively immunosupportive microenvironment, supporting its predictive value to ICI treatment.

Pan-cancer universality of immunotherapy targeting PD-1 and PD-L1 interaction challenges us to rethink the investigation and development of predictive biomarkers. To date, MSI-high (MSI-H) is the only pan-cancer biomarker approved by the FDA with a relatively low frequency $(\sim 4 \%)(33,34)$. MSI-H is common in digestive cancer including colorectal cancer and gastric cancer, while $\mathrm{KDM} 5 \mathrm{C}$ alterations were more common in endometrial and renal cell carcinoma, indicating the predictive value of $\mathrm{MSI}-\mathrm{H}$ and $K D M 5 \mathrm{C}$ alterations is not overlapped. Notably, KDM5C alterations could not predict the clinical outcome in patients with MSS solid tumors receiving ICI, which need future investigation. Collectively, the pan-cancer predictive significance of KDM5C alterations and its complementation to MSI-H in ICI therapy are anticipated.

There are several limitations that should be acknowledged. First, the origin of included cohorts was diverse, which could result in the selection bias and inconsistency of data quality. Combining different groups of patients with distinct histologies without meta-analysis could lead to the methodological pitfalls. Second, the KDM5C altered cohort included both gain (e.g., amplifications) and loss (e.g., deletions) of function alterations whether they could cause the same survival or ICI response difference compared to WT remained undetermined. Without adjustment per histology and type of $K D M 5 C$ alterations, these results should be interpreted with caution. Third, due to the unavailable PDL1 expression results from online database, we could not evaluate the relationship between $K D M 5 C$ alterations and PD-L1 expression. Last but not least, in patients with MSS tumors, only six patients had KDM5C alterations. The association between $K D M 5 C$ alterations and prolonged $O S$ in MSS tumors needs further exploration.

In summary, the present study firstly provides the evidence that $K D M 5 C$ alterations were associated with enhanced tumor immunogenicity and inflamed anti-tumor immunity, which result in prolonged OS in cancer patients treated with ICIs.

\section{REFERENCES}

1. Pardoll DM. The blockade of immune checkpoints in cancer immunotherapy. Nat Rev Cancer (2012) 12:252-64. doi: 10.1038/nrc3239

2. Brahmer JR. Immune checkpoint blockade: the hope for immunotherapy as a treatment of lung cancer? Semin Oncol (2014) 41:126-32. doi: 10.1053/ j.seminoncol.2013.12.014

3. Ribas A, Wolchok JD. Cancer immunotherapy using checkpoint blockade. Science (2018) 359:1350-5. doi: 10.1126/science.aar4060

4. Galluzzi L, Chan TA, Kroemer G, Wolchok JD, Lopez-Soto A. The hallmarks of successful anticancer immunotherapy. Sci Transl Med (2018) 10:eaat7807. doi: 10.1126/scitranslmed.aat7807

5. Sacher AG, Gandhi L. andBiomarkers for the Clinical Use of PD-1/PD-L1 Inhibitors in Non-Small-Cell Lung Cancer: A Review. JAMA Oncol (2016) 2:1217-22. doi: 10.1001/jamaoncol.2016.0639

6. Davoli T, Uno H, Wooten EC, Elledge SJ. Tumor aneuploidy correlates with markers of immune evasion and with reduced response to immunotherapy. Science (2017) 355:eaaf8399. doi: 10.1126/science.aaf8399

7. Chen YP, Zhang Y, Lv JW, Li YQ, Wang YQ, He QM, et al. Genomic Analysis of Tumor Microenvironment Immune Types across 14 Solid Cancer Types:
The predictive value of $K D M 5 C$ alterations were independent of tumor mutational burden and microsatellite status, suggesting that $\mathrm{KDM} 5 \mathrm{C}$ alterations could be considered as a potential pancancer predictive biomarker for ICI treatment. In the future, we still need to investigate the exact molecular mechanism and large-scale, prospective studies are also warranted.

\section{DATA AVAILABILITY STATEMENT}

Publicly available datasets were analyzed in this study. This data can be found here: https://www.cbioportal.org.

\section{ETHICS STATEMENT}

Ethical approval was waived since we used only publicly available data and materials in this study. The patients/participants provided their written informed consent to participate in this study.

\section{AUTHOR CONTRIBUTIONS}

X-JC and LZ designed this study. X-JC and AR collected the clinical and sequenced data. X-JC performed the statistical analyses. X-JC, LZ and E-DZ drafted the manuscript. LZ and E-DZ provided critical comments, suggestions and revised the manuscript. All authors contributed to the article and approved the submitted version.

\section{SUPPLEMENTARY MATERIAL}

The Supplementary Material for this article can be found online at: https://www.frontiersin.org/articles/10.3389/ fimmu.2021.664847/full\#supplementary-material
Immunotherapeutic Implications. Theranostics (2017) 7:3585-94. doi: 10.7150/thno.21471

8. Keenan TE, Burke KP, Van Allen EM. Genomic correlates of response to immune checkpoint blockade. Nat Med (2019) 25:389-402. doi: 10.1038/ s41591-019-0382-x

9. Guo X, Zhang Q. The Emerging Role of Histone Demethylases in Renal Cell Carcinoma. J Kidney Cancer VHL (2017) 4:1-5. doi: 10.15586/jkcvhl.2017.56

10. Wynder C, Stalker L, Doughty ML. Role of H3K4 demethylases in complex neurodevelopmental diseases. Epigenomics (2010) 2:407-18. doi: 10.2217/epi.10.12

11. Monaghan L, Massett ME, Bunschoten RP, Hoose A, Pirvan PA, Liskamp RMJ, et al. The Emerging Role of $\mathrm{H} 3 \mathrm{~K} 9 \mathrm{me} 3$ as a Potential Therapeutic Target in Acute Myeloid Leukemia. Front Oncol (2019) 9:705. doi: 10.3389/ fonc.2019.00705

12. Ji X, Jin S, Qu X, Li K, Wang H, He H, et al. Lysine-specific demethylase $5 \mathrm{C}$ promotes hepatocellular carcinoma cell invasion through inhibition BMP7 expression. BMC Cancer (2015) 15:801. doi: 10.1186/s12885-015-1798-4

13. Hong Z, Wu G, Xiang ZD, Xu CD, Huang SS, Li C, et al. KDM5C is transcriptionally regulated by $\mathrm{BRD} 4$ and promotes castration-resistance prostate cancer cell proliferation by repressing PTEN. BioMed Pharmacother (2019) 114:108793. doi: 10.1016/j.biopha.2019.108793 
14. Lin H, Yang G, Yu J, Wang J, Li Q, Guo S, et al. KDM5c inhibits multidrug resistance of colon cancer cell line by down-regulating ABCC1. BioMed Pharmacother (2018) 107:1205-9. doi: 10.1016/j.biopha.2018.08.041

15. Gao J, Aksoy BA, Dogrusoz U, Dresdner G, Gross B, Sumer SO, et al. Integrative analysis of complex cancer genomics and clinical profiles using the cBioPortal. Sci Signal (2013) 6:pl1. doi: 10.1126/scisignal.2004088

16. Cerami E, Gao J, Dogrusoz U, Gross BE, Sumer SO, Aksoy BA, et al. The cBio cancer genomics portal: an open platform for exploring multidimensional cancer genomics data. Cancer Discovery (2012) 2:401-4. doi: 10.1158/21598290.CD-12-0095

17. Li T, Fan J, Wang B, Traugh N, Chen Q, Liu JS, et al. TIMER: A Web Server for Comprehensive Analysis of Tumor-Infiltrating Immune Cells. Cancer Res (2017) 77:e108-10. doi: 10.1158/0008-5472.CAN-17-0307

18. Li B, Severson E, Pignon JC, Zhao H, Li T, Novak J, et al. Comprehensive analyses of tumor immunity: implications for cancer immunotherapy. Genome Biol (2016) 17:174. doi: 10.1186/s13059-016-1028-7

19. Samstein RM, Lee CH, Shoushtari AN, Hellmann MD, Shen R, Janjigian YY, et al. Tumor mutational load predicts survival after immunotherapy across multiple cancer types. Nat Genet (2019) 51:202-6. doi: 10.1038/s41588-018-0312-8

20. Zehir A, Benayed R, Shah RH, Syed A, Middha S, Kim HR, et al. Mutational landscape of metastatic cancer revealed from prospective clinical sequencing of 10,000 patients. Nat Med (2017) 23:703-13. doi: 10.1038/nm.4333

21. de Cubas AA, Rathmell WK. Epigenetic modifiers: activities in renal cell carcinoma. Nat Rev Urol (2018) 15:599-614. doi: 10.1038/s41585-018-0052-7

22. Chen X, Loo JX, Shi X, Xiong W, Guo Y, Ke H, et al. E6 Protein Expressed by High-Risk HPV Activates Super-Enhancers of the EGFR and c-MET Oncogenes by Destabilizing the Histone Demethylase KDM5C. Cancer Res (2018) 78:1418-30. doi: 10.1158/0008-5472.CAN-17-2118

23. Gossage L, Murtaza M, Slatter AF, Lichtenstein CP, Warren A, Haynes B, et al. Clinical and pathological impact of VHL, PBRM1, BAP1, SETD2, KDM6A, and JARID1c in clear cell renal cell carcinoma. Genes Chromosomes Cancer (2014) 53:38-51. doi: 10.1002/gcc.22116

24. Carlo MI, Manley B, Patil S, Woo KM, Coskey DT, Redzematovic A, et al. Genomic Alterations and Outcomes with VEGF-Targeted Therapy in Patients with Clear Cell Renal Cell Carcinoma. Kidney Cancer (Clifton Va) (2017) 1:49-56. doi: 10.3233/kca-160003

25. Braun DA, Hou Y, Bakouny Z, Ficial M, Sant' Angelo M, Forman J, et al. Interplay of somatic alterations and immune infiltration modulates response to PD-1 blockade in advanced clear cell renal cell carcinoma. Nat Med (2020) 26:909-18. doi: 10.1038/s41591-020-0839-y

26. Dizman N, Lyou Y, Salgia N, Bergerot PG, Hsu J, Enriquez D, et al. Correlates of clinical benefit from immunotherapy and targeted therapy in metastatic renal cell carcinoma: comprehensive genomic and transcriptomic analysis. J Immunother Cancer (2020) 8:e00953. doi: 10.1136/jitc-2020-000953

27. Motzer RJ, Banchereau R, Hamidi H, Powles T, McDermott D, Atkins MB, et al. Molecular Subsets in Renal Cancer Determine Outcome to Checkpoint and Angiogenesis Blockade. Cancer Cell (2020) 38:803-17.e4. doi: 10.1016/ j.ccell.2020.10.011

28. Nassar AH, Hamieh L, Gray KP, Thorner AR, Fay AP, Lasseter KD, et al. Mutations and Response to Rapalogs in Patients with Metastatic Renal Cell Carcinoma. Mol Cancer Ther (2020) 19:690-6. doi: 10.1158/1535-7163.Mct19-0642

29. Lin PH, Huang CY, Yu KJ, Kan HC, Liu CY, Chuang CK, et al. Genomic characterization of clear cell renal cell carcinoma using targeted gene sequencing. Oncol Lett (2021) 21:169. doi: 10.3892/ol.2021.12430

30. Rondinelli B, Schwerer H, Antonini E, Gaviraghi M, Lupi A, Frenquelli M, et al. H3K4me3 demethylation by the histone demethylase KDM5C/JARID1C promotes DNA replication origin firing. Nucleic Acids Res (2015) 43:2560-74. doi: 10.1093/nar/gkv090

31. Rondinelli B, Rosano D, Antonini E, Frenquelli M, Montanini L, Huang D, et al. Histone demethylase JARID1C inactivation triggers genomic instability in sporadic renal cancer. J Clin Invest (2015) 125:4625-37. doi: 10.1172/JCI81040

32. Motzer RJ, Banchereau R, Hamidi H, Powles T, McDermott D, Atkins MB, et al. Molecular Subsets in Renal Cancer Determine Outcome to Checkpoint and Angiogenesis Blockade. Cancer Cell (2020) 38:803-17.e4. doi: 10.1016/ j.ccell.2020.10.011

33. Marcus L, Lemery SJ, Keegan P, Pazdur R. FDA Approval Summary: Pembrolizumab for the Treatment of Microsatellite Instability-High Solid Tumors. Clin Cancer Res (2019) 25:3753-8. doi: 10.1158/1078-0432.CCR-184070

34. Hause RJ, Pritchard CC, Shendure J, Salipante SJ. Classification and characterization of microsatellite instability across 18 cancer types. Nat Med (2016) 22:1342-50. doi: 10.1038/nm.4191

Conflict of Interest: The authors declare that the research was conducted in the absence of any commercial or financial relationships that could be construed as a potential conflict of interest.

Copyright (c) 2021 Chen, Ren, Zheng and Zheng. This is an open-access article distributed under the terms of the Creative Commons Attribution License (CC BY). The use, distribution or reproduction in other forums is permitted, provided the original author(s) and the copyright owner(s) are credited and that the original publication in this journal is cited, in accordance with accepted academic practice. No use, distribution or reproduction is permitted which does not comply with these terms. 